\title{
The decompression of the outer neutron star crust and r-process nucleosynthesis
}

\author{
S. Goriely ${ }^{1}$, N. Chamel ${ }^{1}$, H.-T. Janka ${ }^{2}$, and J. M. Pearson ${ }^{3}$ \\ ${ }^{1}$ Institut d'Astronomie et d'Astrophysique, Université Libre de Bruxelles, CP 226, 1050 Brussels, Belgium \\ e-mail: sgoriely@astro.ulb.ac.be \\ 2 Max-Planck-Institut für Astrophysik, Postfach 1317, 85741 Garching, Germany \\ 3 Département de Physique, Université de Montréal, Montréal (Québec), H3C 3J7, Canada
}

Received 16 March 2011 / Accepted 6 May 2011

\begin{abstract}
Context. The rapid neutron-capture process, or r-process, is known to be fundamental for explaining the origin of approximately half of the $A>60$ stable nuclei observed in nature. In recent years nuclear astrophysicists have developed more and more sophisticated r-process models, by adding new astrophysical or nuclear physics ingredients to explain the solar system composition in a satisfactory way. Despite these efforts, the astrophysical site of the r-process remains unidentified.

Aims. The composition of the neutron star outer crust material is investigated after the decompression that follows its possible ejection. Methods. The composition of the outer crust of a neutron star is estimated before and after decompression. Two different possible initial conditions are considered, namely an idealized crust composed of cold catalyzed matter and a crust initially in nuclear statistical equilibrium at temperatures around $10^{10} \mathrm{~K}$.

Results. We show that in this second case before decompression and at temperatures typically corresponding to $8 \times 10^{9} \mathrm{~K}$, the Coulomb effect owing to the high densities in the crust leads to an overall composition of the outer crust in neutron-rich nuclei with a mass distribution close to the solar system $r$-abundance distribution. Such distributions differ, however, from the solar one due to a systematic shift in the second peak to lower values. After decompression, the capture of the few neutrons per seed nucleus available in the hot outer crust leads to a final distribution of stable neutron-rich nuclei with a mass distribution of $80 \leq A \leq 140$ nuclei in excellent agreement with the solar distribution, provided the outer crust is initially at temperatures around $8 \times 10^{9} \mathrm{~K}$ and all layers of the outer crust are ejected.

Conclusions. The decompression of the neutron star matter from the outer crust provides suitable conditions for a robust r-processing of the light species, i.e., r-nuclei with $A \leq 140$. The final composition should carry the imprint of the temperature at which the nuclear statistical equilibrium is frozen prior to the ejection.
\end{abstract}

Key words. nuclear reactions, nucleosynthesis, abundances - stars: neutron - equation of state

\section{Introduction}

The r-process, or the rapid neutron-capture process, of stellar nucleosynthesis is invoked to explain the production of the stable (and some long-lived radioactive) neutron-rich nuclides that are heavier than iron and observed in stars of various metallicities, as well as in the solar system (for a review, see Arnould et al. 2007). In recent years nuclear astrophysicists have developed more and more sophisticated r-process models, trying to explain the solar system composition in a satisfactory way by adding new astrophysical or nuclear physics ingredients. The r-process remains the most complex nucleosynthetic process for modeling from the astrophysics as well as nuclear-physics points of view. The site(s) of the r-process has (have) not been identified yet, since all the proposed scenarios face serious problems. Complex - and often exotic - sites have been considered in the hope of identifying astrophysical conditions in which the production of neutrons is high enough to give rise to a successful r-process.

Progress in the modeling of type-II supernovae and $\gamma$-ray bursts has raised a lot of excitement about the so-called neutrinodriven wind environment. However, until now a successful rprocess cannot be obtained ab initio without tuning the relevant parameters (neutron excess, entropy, expansion timescale) in a way that is not supported by the most sophisticated existing models. Although these scenarios remain promising, especially in view of their potential to significantly contribute to the galactic enrichment (Argast et al. 2004), they remain handicapped by large uncertainties associated mainly with the still incompletely understood mechanism that is responsible for the supernova explosion and the persistent difficulties obtaining suitable r-process conditions in self-consistent dynamical explosion and neutronstar cooling models (Hüdepohl et al. 2010; Fischer et al. 2010). In addition, predictions of the detailed composition of the ejected matter remain difficult owing to the remarkable sensitivity of $r$ process nucleosynthesis to uncertainties of the ejecta properties.

Early in the development of the theory of nucleosynthesis, an alternative to the r-process in high-temperature supernova environments was proposed (Tsuruta et al. 1965). It relies on the tendency of matter at high densities (typically $\rho>10^{10} \mathrm{~g} \mathrm{~cm}^{-3}$ ) to be composed of nuclei lying on the neutron-rich side of the valley of nuclear stability as a result of endothermic free-electron captures. This so-called "neutronization" of matter is possible even at zero temperature. The astrophysical plausibility of this scenario in accounting for the production of the r-nuclides has long been questioned. It remained largely unexplored until the study of the decompression of cold neutronized matter resulting from tidal effects of a black hole on a neutron star (NS) companion (Lattimer et al. 1977; Meyer 1989). Recently, special 
attention has been paid to NS mergers following the confirmation by hydrodynamic simulations that a non-negligible amount of matter can be ejected (Janka et al. 1999; Rosswog et al. 2004; Oechslin et al. 2007). The ejection of initially cold, decompressed NS matter might also happen in other astrophysical scenarios like giant flares in soft-gamma repeaters, the explosion of an NS eroded below its minimum mass (e.g. Sumiyoshi et al. 1998), or the equatorial shedding of material from very rapidly rotating supramassive or ultramassive NSs (see Arnould et al. 2007, for more details).

The composition of the inner crust, before and after decompression, has already been studied in detail in Goriely et al. (2005) and Arnould et al. (2007). It was found that the final composition of the material ejected from the inner crust is expected to depend on the initial density, at least for the upper part of the inner crust at $\rho_{\text {drip }} \leq \rho\left[\mathrm{g} / \mathrm{cm}^{3}\right] \leq 10^{12}$ (where $\rho_{\text {drip }} \simeq 4.2 \times 10^{11} \mathrm{~g} / \mathrm{cm}^{3}$ is the neutron drip density). For the deeper inner-crust layers $\left(\rho>10^{12} \mathrm{~g} / \mathrm{cm}^{3}\right)$, large neutron-toseed ratios drive the nuclear flow into the very heavy mass region, leading to fission recycling. As a consequence, the abundance distribution is now independent of the initial conditions, especially the initial density. In both cases, the abundance distribution was found to be in close agreement with the solar distribution for $A>130$ nuclei (Goriely et al. 2005; Arnould et al. 2007).

Although the outer crust is far less massive than the inner crust, the ejection of the inner crust cannot take place without at the same time leading to the ejection of at least some outer crust material. The outer crust typically amounts to $10^{-5}$ to $10^{-4} M_{\odot}$, depending on the NS mass and radius (Pearson et al. 2011) so that depending on the frequency and fraction of its possible ejection, it may or may not contribute significantly to the galactic enrichment. We will not get into these considerations in the present paper, but restrict ourselves to estimating the composition of the outer crust material before (Sect. 2) as well as after ejection (Sect. 3), assuming that nuclear statistical equilibrium (NSE) could be established before ejection occurred. We shall consider two cases: the first one corresponds to an initially cold NS crust and the second one to an NS crust initially in NSE at a temperature of the order of $10^{10} \mathrm{~K}$ at which nuclear statistical equilibrium (NSE) is responsible for setting the composition prior to the ejection.

\section{Composition of the NS outer crust in equilibrium}

The composition of the outer crust needs to be estimated in each layer, each one being characterized by a given density and pressure. The overall abundance distribution for the outer crust $\left(0 \leq \rho(r) \leq \rho_{\text {drip }}\right)$ can then be integrated over a pressure column $P(r)$ (where $r$ is the radial coordinate) given by the Tolman-Oppenheimer-Volkoff(TOV) equations (considering non-rotating NS)

$\frac{\mathrm{d} P}{\mathrm{~d} r}=-G \frac{\left\{\mathcal{M}(r)+4 \pi r^{3} P(r) / c^{2}\right\}\{\mathcal{E}(r)+P(r)\}}{r\left\{r c^{2}-2 G \mathcal{M}(r)\right\}}$

with

$\frac{\mathrm{d} \mathcal{M}}{\mathrm{d} r}=4 \pi \mathcal{E}(r) r^{2} / c^{2}$,

in which $\mathcal{E}$ denotes the total energy density, including rest mass (in the outer crust, $\mathcal{E} \simeq \rho c^{2}$ ) and $\mathcal{M}$ is the gravitational mass. We choose here a standard NS with a mass of $1.5 M_{\odot}$ and $13 \mathrm{~km}$ radius. Since the equation of state $(\mathrm{EoS})$ and the composition remain sensitive to the nuclear physics ingredients adopted (Pearson et al. 2011), we will consider here three different Hartree-Fock-Bogolyubov (HFB) mass models, namely the Skyrme-HFB mass models HFB-19 and HFB-21 (Goriely et al. 2010) and the Gogny-HFB mass model D1M (Goriely et al. 2009). For each mass model the EoS has been consistently taken into account in solving the TOV equation. In the three cases, a baryonic mass of about $5 \times 10^{-5} M_{\odot}$ (Pearson et al. 2011) is found in the outer crust of such an NS and about $90 \%$ of the outer-crust mass is located in the density range of $6 \times 10^{10} \mathrm{~g} / \mathrm{cm}^{3} \lesssim \rho \leq \rho_{\text {drip }}$. For densities above the drip density, free neutrons are present and their contributions to the EoS should be included. A transitional regime into the inner crust can be included in the calculation by estimating the pressure and free energy associated with the corresponding gas of free neutrons obtained self-consistently with the effective interactions BSk19, BSk21 or D1M corresponding to the above-mentioned mass tables.

\subsection{The cold NS crust}

A fairly common picture of an NS (Baym et al. 1971; Pethick \& Ravenhall 1995) is that they consist of "cold catalyzed matter", i.e., electrically neutral matter in its absolute ground state in the sense of complete nuclear and beta equilibrium at temperature $T=0$. Detailed calculations of the composition of the NS material initially at a density below the drip density $\left(\rho_{\text {drip }}=4.2 \times 10^{11} \mathrm{~g} \mathrm{~cm}^{-3}\right)$ have been performed over the years (Baym et al. 1971; Pethick \& Ravenhall 1995; Haensel \& Pichon 1994; Rüster et al. 2006; Pearson et al. 2011). We have repeated the calculation of Baym et al. (1971) with the updated nuclear physics inputs to determine the outer-crust composition. This calculation minimizes the free Gibbs energy per nucleon at $T=0$. All details can be found in Pearson et al. (2011) and will not be repeated here.

For densities above $3 \times 10^{9} \mathrm{~g} / \mathrm{cm}^{3}$, the outer crust is essentially made of $N=50$ and $N=82$ neutron-rich nuclei. More precisely, for $10^{9} \leq \rho\left[\mathrm{g} / \mathrm{cm}^{3}\right] \lesssim 5 \times 10^{10}$, we find $N=50$ nuclei with $80 \leq A \leq 86$. At these densities, only nuclei with experimentally known masses are involved. This is not the case at higher densities, i.e., in the most massive part of the outer crust, where we use the HFB-19, HFB-21 or D1M mass tables to complement experimental masses. For $10^{11} \leq \rho\left[\mathrm{g} / \mathrm{cm}^{3}\right] \leq \rho_{\text {drip }}$, $N=82$ nuclei with $120 \leq A \leq 126$ populate the outer crust.

The variation of the $T=0$ composition of the outer crust as a function of density is illustrated in Fig. 1. In this density range, the electron fraction $Y_{\mathrm{e}}$ varies from 0.43 down to 0.31 at the drip density. Once in possession of the solution $\rho(r)$ to the TOV equations, it is easy to calculate the baryonic mass of any shell whose inner and outer radii $r_{1}$ and $r_{2}$, respectively, are chosen to correspond to given densities $\rho\left(r_{1}\right)$ and $\rho\left(r_{2}\right)$ (Pearson et al. 2011). The abundances of the different nuclides in the outer crust can then be read off from Fig. 1, the results being shown in Fig. 2. Slightly different distributions are obtained with different mass models, but generally, because of the high mass included within the high-density region (close to the drip point), nuclides with $A \simeq 80$ or $118 \lesssim A \lesssim 126$ will be dominant, as can be seen in Fig. 2.

If a decompression of the $T=0$ outer crust material takes place, only $\beta$-decays towards the valley of stability are expected, no free neutrons being present (except through the possible $\beta$ delayed neutron emission). Consequently, after decompression 
S. Goriely et al.: The r-process nucleosynthesis in neutron stars

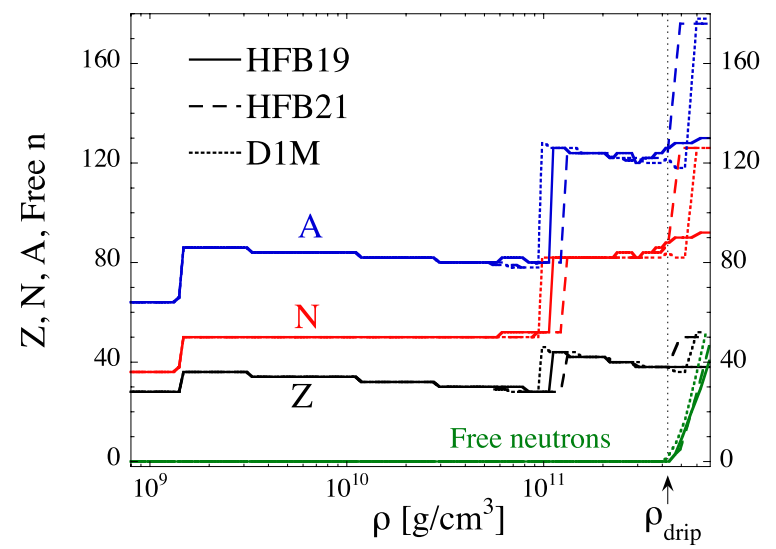

Fig. 1. Ground-state composition (charge, neutron and mass numbers as well as free neutrons) of the outer crust and of the shallow layers of the inner crust as a function of the density. Predictions with HFB-19 masses (solid lines) (Goriely et al. 2010) are compared with those obtained with the D1M masses (dotted lines) (Goriely et al. 2009). Experimental masses (Audi et al. 2003, 2010) are used whenever available.

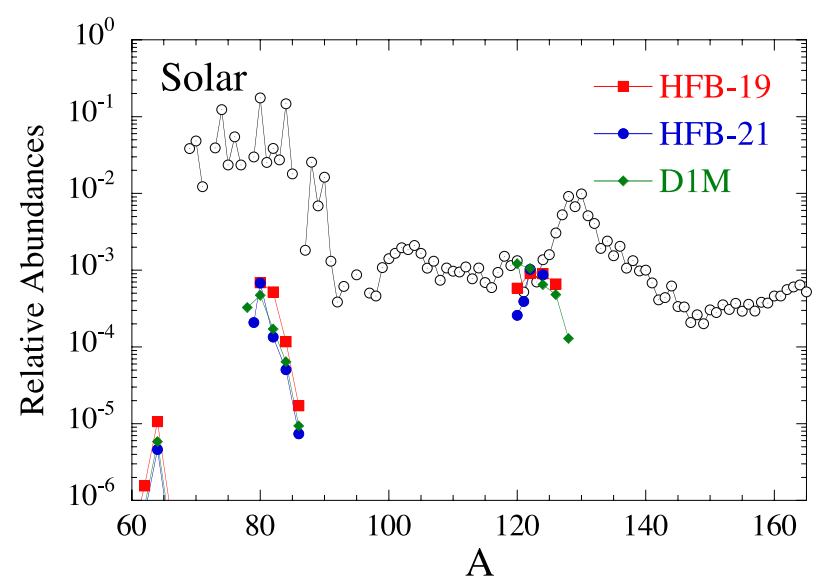

Fig. 2. Abundance distribution integrated over the whole outer crust for an initially cold NS of mass $1.5 M_{\odot}$ and $13 \mathrm{~km}$ radius. The distributions are arbitrarily normalized to the solar system $\mathrm{r}$-abundance distribution, shown for comparison. The distributions are obtained with the HFB-19 (squares), HFB-21 (circles) and D1M (diamonds) mass models when experimental masses (Audi et al. 2003, 2010) are not available.

only a restricted distribution of $78 \lesssim A \lesssim 86$ and $120 \lesssim A \lesssim 126$ nuclei can be expected, as discussed in Sect. 3.1.

\subsection{The hot NS crust}

The $T=0$ picture of the NS crust is clearly an idealization, as it implies that the star has had an infinite time to cool down and maintain (or restore) thermodynamic equilibrium since its creation in the aftermath of a gravitational-collapse supernova explosion. In reality, not only will the actual temperature of any particular NS be non-zero, but the equilibrium configuration corresponding to a still higher temperature might have become "frozen in". The outer NS crust may in fact have a very different composition at non-zero temperature due to the specific softness of the distribution of the Gibbs free energy per nucleon.

As shown in Fig. 3, many nuclei yield a free energy per nucleon that differs from the equilibrium one by no more than a few tens of $\mathrm{keV}$. At finite temperatures, thermal fluctuations could therefore significantly broaden the distribution of equilibrium nuclides. At $T_{9} \lesssim 4-5$ (where $T_{9}$ is the temperature expressed

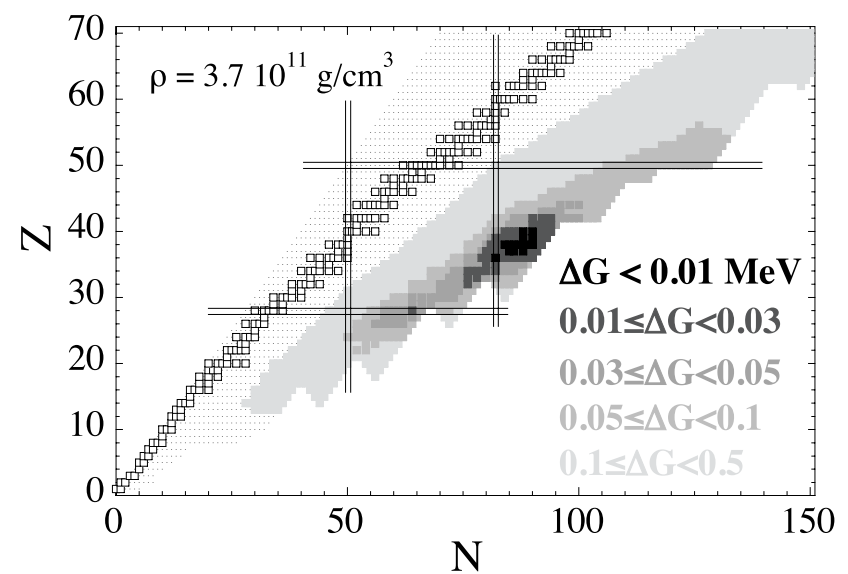

Fig. 3. Distribution of the Gibbs free energy per nucleon at $T=0$ and $\rho=3.7 \times 10^{11} \mathrm{~g} / \mathrm{cm}^{3} . \Delta G$ corresponds to the difference of the free energy per nucleon with respect to the minimum value obtained at this density for $Z=38, N=88$ and $A=124$. The distribution is obtained with the HFB-19 mass model (Goriely et al. 2010) when experimental masses are not available. The open squares represent stable nuclei.

in $\left.10^{9} \mathrm{~K}\right)$, an $(n, \gamma)-(\gamma, n)$ equilibrium may be established within the isotopic chain corresponding to the most probable $Z$ value at the given density. For higher temperatures, an NSE could be reached and the abundance of a given nucleus $i$ of $Z$ protons and $N$ neutrons $(A=Z+N)$ is now given by (Bravo \& Garcia-Senz 1999; Nadyoshin \& Yudin 2005; Arcones et al. 2010)

$Y_{i}=\frac{\omega_{i}}{\rho / m_{u}}\left(\frac{k T A m_{u}}{2 \pi \hbar^{2}}\right)^{3 / 2} \mathrm{e}^{N \eta_{n}+Z \eta_{p}} \mathrm{e}^{Q_{i} / k T}$

where $\omega_{i}$ is the $T$-dependent partition function, and

$Q_{i}=\left[Z m_{\mathrm{p}}+N m_{\mathrm{n}}-m_{i}\right] c^{2}+Z \mu_{\mathrm{C}, \mathrm{p}}-\mu_{\mathrm{C}}(Z, A)$,

includes the binding energy, expressed in term of the nucleon and nuclear masses $M_{i}$, as well as the Coulomb corrections to the chemical potential $\mu_{\mathrm{C}}$ arising from the Coulomb contribution to the free energy, which becomes significant for heavier nuclei. Equation (3) assumes that nuclei follow Maxwell-Boltzmann statistics, but the presence of the degeneracy parameters $\eta_{\mathrm{n}}$ and $\eta_{\mathrm{p}}$ takes into account the possibility that nucleons follow the more general Fermi-Dirac statistics, which will certainly be the case for free neutrons close to the drip density. These parameters are related to the number density $n_{q}$ through the usual expression

$n_{q}=\frac{8 \pi \sqrt{2}}{h^{3}} m_{q}^{3} c^{3} \beta_{q}^{3}\left[\mathcal{F}_{1 / 2}\left(\eta_{q}, \beta_{q}\right)+\beta_{q} \mathcal{F}_{3 / 2}\left(\eta_{q}, \beta_{q}\right)\right]$,

where $\beta=k T / m c^{2}$ is the relativistic parameter and $\mathcal{F}_{k}$ are the Fermi functions

$\mathcal{F}_{k}(\eta, \beta)=\int_{0}^{\infty} \frac{x^{k}(1+\beta x / 2)^{1 / 2}}{\mathrm{e}^{-\eta+x}+1} \mathrm{~d} x$.

More details on the high-density NSE equations can be found in Bravo \& Garcia-Senz (1999) and Arcones et al. (2010).

To estimate the composition of the hot crust, the same EoS is used as in Pearson et al. (2011), though the T-dependent nonideal Coulomb interaction between ions as well as between ions and electrons and between electrons in the approximation of a rigid electron background is taken from Haensel et al. (2007) 


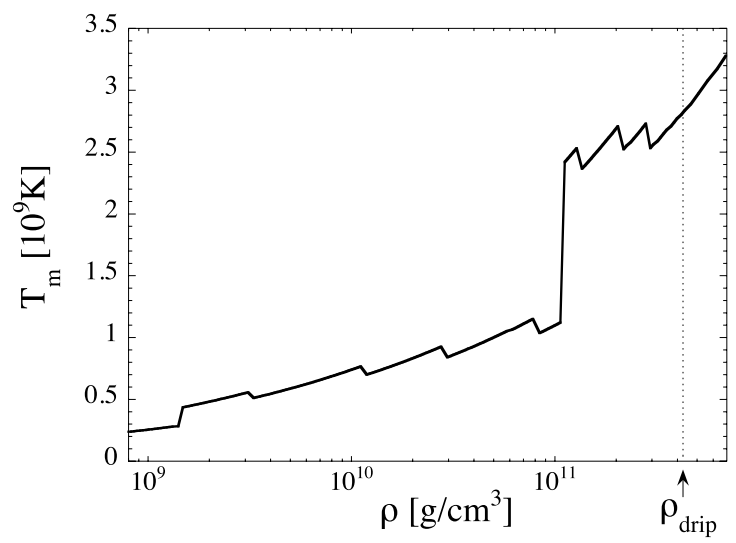

Fig. 4. Melting temperature $T_{\mathrm{m}}$ as a function of the density $\rho$, estimated from Eq. (10) and the $T=0$ composition shown in Fig. 1 using HFB-19 masses when no experimental masses are available.

and DeWitt et al. (1996). In this approximation, the Coulomb chemical potential of species $i$ is given by

$\mu_{\mathrm{C}}(Z, A)=k T f_{\mathrm{C}}\left(\Gamma_{i}\right)$

where $f_{\mathrm{C}}$ is the Coulomb free energy per ion in units of $k T$. We would like to point out that we did not include here the contribution of $k T / 3 \times \partial f_{\mathrm{C}} / \partial \ln \Gamma_{\mathrm{i}}$ as proposed by Glazyrin \& Blinnikov (2010), since this contribution implies a variation of the electron density and should therefore not be included in the NSE equations which are calculated at a fixed value of the electron density (see the discussion p. 4 of Glazyrin \& Blinnikov 2010).

For a Coulomb liquid, $f_{\mathrm{C}}$ can be expressed as (Haensel et al. 2007)

$$
\begin{aligned}
f_{\mathrm{C}}= & \frac{F_{\mathrm{C}}}{n_{\mathrm{i}} k T}=A_{1} \sqrt{\Gamma_{\mathrm{i}}\left(A_{2}+\Gamma_{\mathrm{i}}\right)} \\
& -A_{1} \times A_{2} \ln \left(\sqrt{\Gamma_{\mathrm{i}} / A_{2}}+\sqrt{1+\Gamma_{\mathrm{i}} / A_{2}}\right) \\
& +2 A_{3}\left[\sqrt{\Gamma_{\mathrm{i}}}-\arctan \left(\sqrt{\Gamma_{\mathrm{i}}}\right)\right] \\
& +B_{1}\left[\Gamma_{\mathrm{i}}-B_{2} \ln \left(1+\frac{\Gamma_{\mathrm{i}}}{B_{2}}\right)\right]+\frac{B_{3}}{2} \ln \left(1+\frac{\Gamma_{\mathrm{i}}^{2}}{B_{4}}\right),
\end{aligned}
$$

with $A_{1}=-0.9070, A_{2}=0.62954, A_{3}=0.27710, B_{1}=$ $0.00456, B_{2}=211.6, B_{3}=-0.0001$ and $B_{4}=0.00462$. The corresponding contribution to the internal energy and the pressure can be found in Haensel et al. (2007).

The Coulomb liquid approximation is adopted since at the temperatures considered here (typically $T_{9}=5-10$ ), the Coulomb coupling parameter,

$\Gamma_{\mathrm{i}}=\frac{Z^{2} \mathrm{e}^{2}}{a_{\mathrm{i}} k T}$

where $a_{\mathrm{i}}$ is the ion-sphere radius, remains smaller than the melting value $\Gamma_{\mathrm{m}}=175.0 \pm 0.4$ (Pothekin \& Chabrier 2000). Equivalently, the temperature $T$ is higher than the melting temperature,

$T_{\mathrm{m}}=\frac{Z^{2} \mathrm{e}^{2}}{a_{\mathrm{i}} k \Gamma_{\mathrm{m}}} \simeq 1.3 \times 10^{3} Z^{2}\left(\frac{\rho\left[\mathrm{g} / \mathrm{cm}^{3}\right]}{A}\right)^{1 / 3} \mathrm{~K}$,

as shown in Fig. 4, where $T_{\mathrm{m}}$ has been evaluated on the basis of the $T=0$ composition given in Fig. 1 (based on HFB-19 masses).

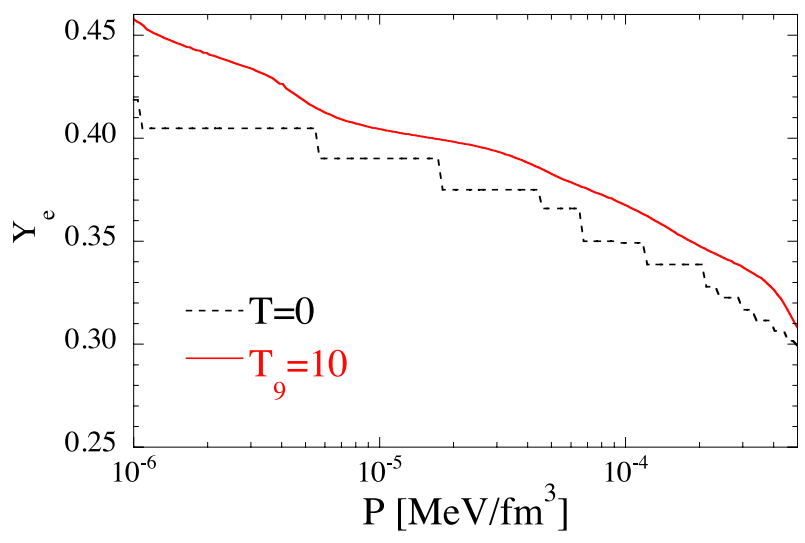

Fig. 5. $Y_{\mathrm{e}}$ distribution in the outer crust, assuming (neutrino-less) $\beta$ equilibrium, as a function of the pressure. The dashed line corresponds to $T=0$ and the solid line to $T=10^{10} \mathrm{~K}$.

To estimate the NSE composition of a hot NS crust still requires the determination of the distribution of the electron fraction $Y_{\mathrm{e}}$, i.e., its variation with the density $n$. We assume here that the NS cooled sufficiently slowly for $\beta$-equilibrium to be maintained down to a temperature of about $T \simeq 10^{10} \mathrm{~K}$, at which point the $Y_{\mathrm{e}}$ distribution becomes frozen in as the NS continues to cool to lower temperatures. However, we assume that NSE is maintained down to temperatures of the order of $T \simeq 7$ $10 \times 10^{9} \mathrm{~K}$ (see Sect. 3.2 for a discussion of the NSE timescales). The $Y_{\mathrm{e}}$ distribution corresponding to $\beta$-equilibrium is obtained by minimizing the free Gibbs energy per nucleon at $T=10^{10} \mathrm{~K}$; for the physics used, see also Pearson et al. (2011). We use the traditional linear mixing rule (also known as the additive approximation) to estimate the total free Gibbs energy of the multicomponent plasma by weighting the individual free energy of each species by its molar fraction in the mixture. With respect to the $T=0$ case, the $Y_{\mathrm{e}}$ distribution is found to be slightly higher, and obviously much smoother, as shown in Fig. 5. The implied decrease in the neutron fraction occurs despite the liberation of free neutrons at non-zero temperatures.

The NSE abundance distributions for the whole outer crust at four different temperatures (between 7 and $10 \mathrm{GK}$ ) and integrated over a pressure column given by the TOV equations are shown in Fig. 6. The outer NS crust in NSE at a temperature of $T_{9} \simeq 7-10$ is seen to be made of r-nuclei with a distribution similar to that of the solar system, although the second peak is slightly shifted to lower masses around $A \simeq 126$, as is also found for the $T=0$ composition (Fig. 2). The second peak is also found to be rather pronounced with respect to the first peak for low temperatures $\left(T_{9}=7\right)$, but to be smoothed away, on the other hand, for high temperatures $\left(T_{9}=10\right)$. The abundance distribution is sensitive to the adopted mass models, as illustrated in Fig. 7, but is otherwise extremely robust, since as far as the nuclear physics is concerned it depends only on binding energies. The Coulomb correction plays a vital role by shifting significantly the abundance distribution towards the high-mass region. In particular, no $N \simeq 82$ would be found in the crust if the Coulomb interaction was omitted in the NSE equations, as shown in Fig. 8.

\section{Decompression of the NS outer crust}

Without focussing clearly on a particular ejection scenario of outer-crust material of an NS, we describe the density evolution of an ejecta clump in simple, but as general terms as possible. For 


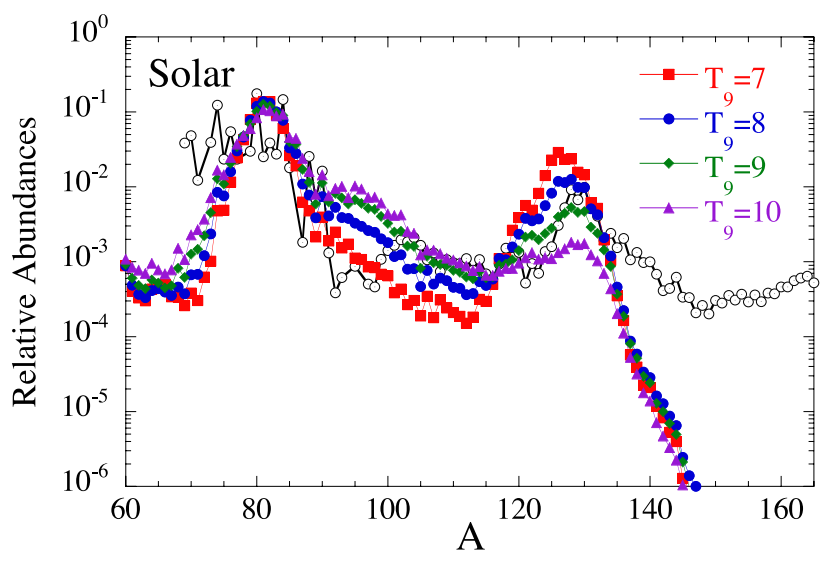

Fig. 6. NSE abundance distribution integrated over the whole outer crust for 4 different temperatures assuming the initial $Y_{\mathrm{e}}$ distribution is determined by $\beta$-equilibrium at $T=10^{10} \mathrm{~K}$. The distribution is obtained with the HFB-19 mass model (Goriely et al. 2010) when experimental masses are not available. The solar system $\mathrm{r}$-abundance distribution (dotted circles) is shown for comparison.

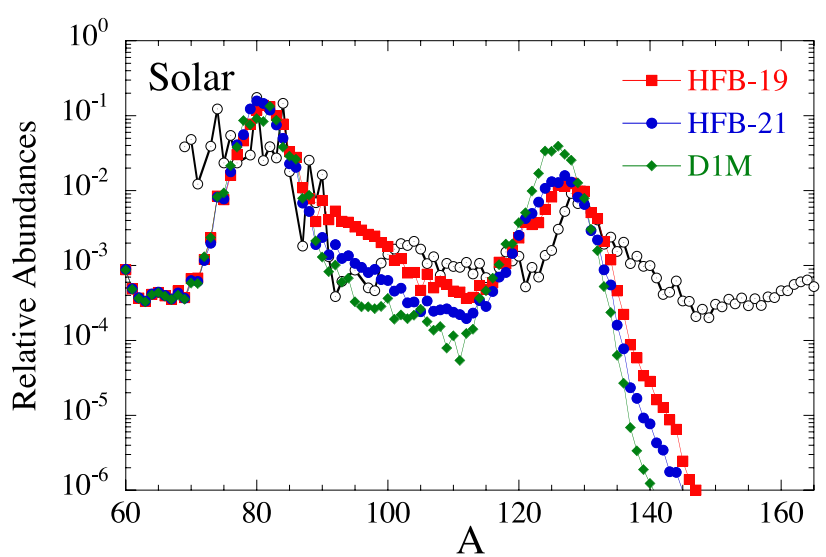

Fig. 7. Same as Fig. 6 for $T_{9}=8$ and different mass models, namely HFB-19, HFB-21 (Goriely et al. 2010) or D1M (Goriely et al. 2009).

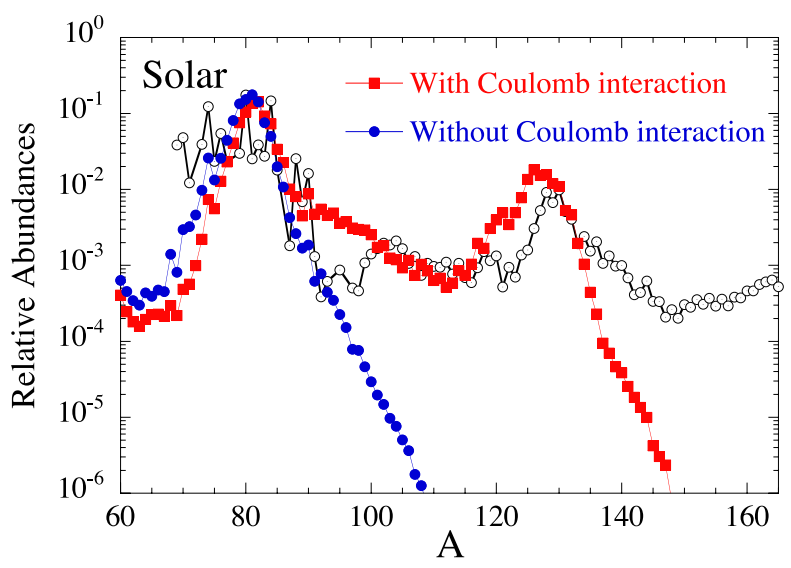

Fig. 8. Same as Fig. 7 including and excluding the Coulomb correction to the free energy (the HFB-19 mass model is used).

setting the values of the free parameters we will refer to existing results from simulations of the dynamical merging of binary NSs or an NS with a black hole. In these events near-surface matter can be tidally stripped and ejected, and we take the ejection dynamics of such events as template for our expansion model.
The evolution of the matter density is approximated by a onezone model of the pressure-driven expansion of a self-gravitating clump under the influence of tidal forces (exerted by the compact source object of the ejecta) along the escape trajectory of the clump. In order to determine the time evolution of the clump radius $R(t)$, we integrate numerically the Newtonian equation of motion

$\frac{\mathrm{d}^{2} R}{\mathrm{~d} t^{2}}=-\frac{4 \pi}{3} G \rho R+\frac{P}{\rho R}+\frac{R}{\left(\tau_{\mathrm{esc}}+\frac{3}{2} t\right)^{2}}$.

Here the first term corresponds to the confining acceleration by the self-gravity of the clump, approximated by $-G M_{\mathrm{c}} / R^{2} \approx$ $-\frac{4 \pi}{3} G \rho R$ when $M_{\mathrm{c}} \approx \frac{4 \pi}{3} \rho R^{3}$ is the clump mass with average density $\rho$. The inflating effect of the gas pressure $P$ of the clump is repesented by the second term in Eq. (11), where we use a one-zone representation of the pressure gradient according to $\rho^{-1} \mathrm{~d} P / \mathrm{d} r \approx P /(\rho R)$. The third term describes the acceleration corresponding to the tidal stretching by the external gravitational force of the NS,

$\left(\frac{\mathrm{d}^{2} R}{\mathrm{~d} t^{2}}\right)_{\text {tidal }} \sim \frac{G M_{\mathrm{ns}}}{r_{\mathrm{c}}^{2}}-\frac{G M_{\mathrm{ns}}}{\left(r_{\mathrm{c}}+R\right)^{2}} \approx \frac{2 G M_{\mathrm{ns}} R}{r_{\mathrm{c}}^{3}}$,

where $M_{\mathrm{ns}}$ is the NS mass and $r_{\mathrm{c}}(t)$ the time-dependent radial position of the clump center. In the transformation leading to the last term in Eq. (12) we made use of the relation $R \ll r_{\mathrm{c}}$. Assuming that the ejection velocity $v_{\mathrm{c}}=\mathrm{d} r_{\mathrm{c}} / \mathrm{d} t$ of the clump is given by the escape velocity on a parabolic orbit, i.e., $\frac{1}{2} v_{\mathrm{c}}^{2}=G M_{\mathrm{ns}} / r_{\mathrm{c}}$, we can solve the equation of motion of the clump center, $\mathrm{d} r_{\mathrm{c}} / \mathrm{d} t=\sqrt{2 G M_{\mathrm{ns}} / r_{\mathrm{c}}}$, to obtain

$r_{\mathrm{c}}(t)=r_{0}\left(1+\frac{3}{2} \frac{t}{\tau_{\mathrm{esc}}}\right)^{2 / 3}$,

with $r_{0}$ being the radial position of the clump center at the beginning of the ejection, $\tau_{\text {esc }}=r_{0} / v_{\text {esc }}$ the escape timescale, and $v_{\mathrm{esc}}=\sqrt{2 G M_{\mathrm{ns}} / r_{0}}$ the escape velocity. Using $r_{\mathrm{c}}(t)$ of Eqs. (13) in (12) we get

$\left(\frac{\mathrm{d}^{2} R}{\mathrm{~d} t^{2}}\right)_{\text {tidal }} \sim \frac{R}{\left(\tau_{\mathrm{esc}}+\frac{3}{2} t\right)^{2}}$,

which appears as the last term on the rhs of Eq. (11). Analytic estimates for the conditions at the surface of an NS in agreement with numerical results of NS merger simulations (Ruffert $\&$ Janka 2001) yield values of the escape timescale $\tau_{\text {esc }}$ in the range of $10^{-4}$ to $3 \times 10^{-4} \mathrm{~s}$. The value of $\tau_{\text {esc }}=3 \times 10^{-4} \mathrm{~s}$ is adopted in this work.

In solving the simple model equation, Eq. (11), the initial clump radius $R_{0}$ plays an important role, because it determines the initial acceleration of the clump expansion and therefore its expansion timescale. It can be used as a free parameter, leading to different density decline rates during decompression. Here we choose a value of $R_{0} \approx 2 \mathrm{~km}$, which is found to reproduce the density evolution of the majority of NS merger ejecta (Ruffert $\&$ Janka 2001) fairly well. The pressure $P$ of the clump medium is determined from the EoS of Timmes \& Arnett (1999) and is consistently evolved for the changing conditions of density and temperature (including possible $\beta$-decay heating) during the decompression history. The integration of Eq. (11) yields the timedependent clump radius $R(t)$, with which (for constant clump mass) the clump density can be computed as $\rho(t)=\rho_{0}\left(R_{0} / R(t)\right)^{3}$. 


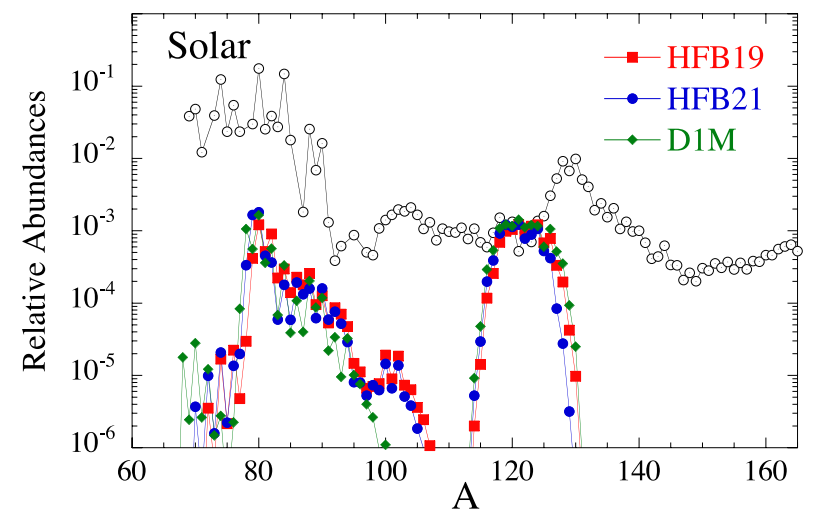

Fig. 9. Same as Fig. 2 after decompression.

The composition change during the decompression is followed with a full network, where in particular the $\beta$-decay processes have been taken from the updated version of the Gross Theory (Tachibana et al. 1990) and neutron capture rates are consistently estimated with the TALYS code (Goriely et al. 2008) on the basis of the nuclear mass model used for the initial conditions. The temperature evolution is followed as described in Meyer (1989) and Goriely et al. (2005) on the basis of the laws of thermodynamics and possible nuclear heating through $\beta$-decay processes. The decompression along the above-defined trajectory of both the cold NS outer crust and the outer crust initially in NSE are studied below.

\subsection{The cold NS crust}

The final isobaric abundance distribution of the matter ejected from the cold NS crust initially assumed to be at $\beta$-equilibrium can be expected to remain relatively similar to the one prior to the ejection, since only $\beta$-decay and $\beta$-delayed neutron emission (with the possibility of recapturing the emitted neutrons) may change the initial composition. The final isobaric abundance distribution is shown in Fig. 9, the initial composition being given in Fig. 2 prior to the decompression. Globally, the final distribution is obviously far from matching the overall solar system pattern. Some differences are seen depending on the nuclear physics ingredients adopted. Interestingly, the decompression of the cold NS outer crust appears as a potential site for producing almost exclusively the $115 \lesssim A \lesssim 124$ r-nuclei. In some siteindependent parametrized r-process models, these nuclei have been found to be underproduced, though this conclusion is obviously subject to large nuclear physics and astrophysics uncertainties (for a review, see Arnould et al. 2007).

\subsection{The hot NS crust}

As discussed in Sect. 2.2, the outer crust initially in NSE at temperatures around $T_{9}=7-10$ differs considerably from the cold $T=0$ case. In addition, for layers close to the drip density, free neutrons are present in significant numbers due to the high initial temperatures. In this case, a few neutrons per seed nucleus are available and may be captured during the expansion phase so that the initial NSE mass distribution is modified. The abundance distributions in a layer with initial pressure $P_{0}=3 \times 10^{-4} \mathrm{MeV} / \mathrm{fm}^{3}$ and density $\rho_{0}=2.7 \times 10^{11} \mathrm{~g} / \mathrm{cm}^{3}$, initially in NSE at $T_{9}=8$ is shown in Fig. 10. The evolution of the temperature, density as well as the corresponding radioactive power due to $\beta$-decays is shown in Fig. 11 for the same ejected layer as the one considered

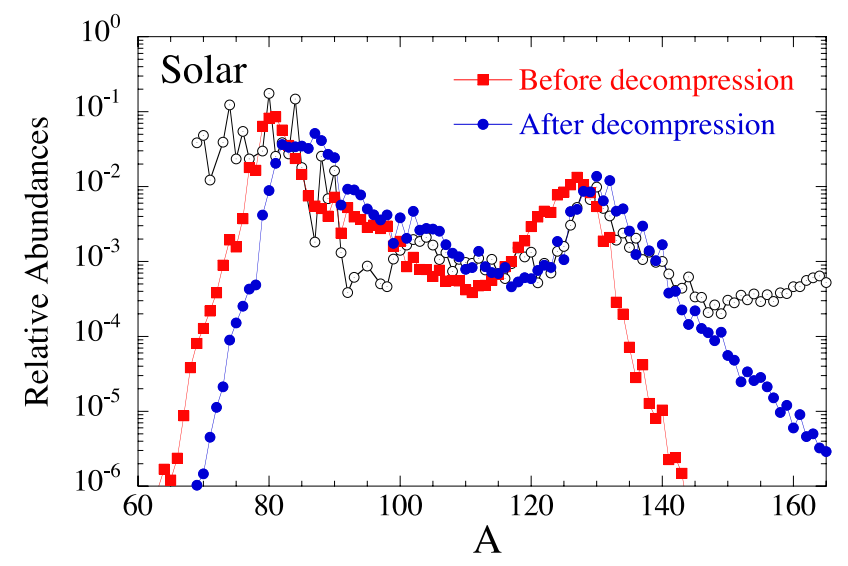

Fig. 10. Abundance distribution before and after decompression of a layer with initial pressure $P_{0}=4 \times 10^{-4} \mathrm{MeV} / \mathrm{fm}^{3}$ and density $\rho_{0}=$ $3.4 \times 10^{11} \mathrm{~g} / \mathrm{cm}^{3}$ and initially in NSE at $T_{9}=8$. The calculation was performed with the HFB-19 masses and corresponding reaction rates.

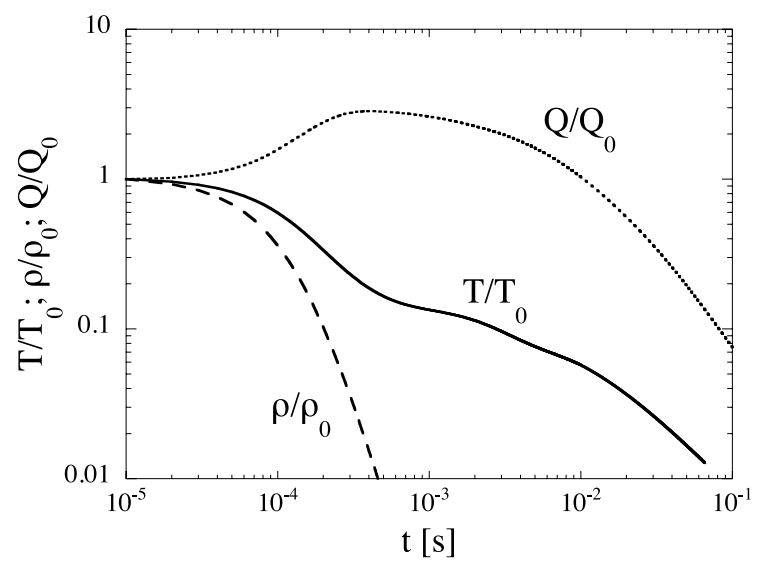

Fig. 11. Evolution of the temperature, density and radioactive heating rate per unit mass $(Q)$ resulting from the decompression of the layer with an initial pressure $P_{0}=4 \times 10^{-4} \mathrm{MeV} / \mathrm{fm}^{3}$ and density $\rho_{0}=$ $3.4 \times 10^{11} \mathrm{~g} / \mathrm{cm}^{3}$ and initially in NSE at $T_{0}=8 \times 10^{9} \mathrm{~K}$. The initial radioactive decay heat $Q_{0}$ amounts to $6.6 \times 10^{18} \mathrm{erg} \mathrm{g}^{-1} \mathrm{~s}^{-1}$.

in Fig. 10. In particular, it can be seen that the $\beta$-decay heating slows down the temperature drop already a few ms after ejection.

After decompression, due to the capture of about 5 free neutrons per seed nucleus, the distribution is shifted towards the $A \simeq 130$ peak and shaped by the lower temperatures found at the time of the neutron captures. The final distribution is found to be independent of the details characterizing the expansion. In particular for a faster expansion obtained with the value of $\tau_{\text {esc }}=10^{-4} \mathrm{~s}$ a virtually identical distribution is found.

The abundance pattern for the outer crust integrated up to a maximum pressure $P$ is given in Fig. 12 for the initial temperature of $T_{9}=8$. If the whole outer crust is considered $\left(P \leq P_{\text {drip }}=5 \times 10^{-4} \mathrm{MeV} / \mathrm{fm}^{3}\right)$, an overall agreement with the solar distribution is obtained in the whole $80 \lesssim A \lesssim 150$ mass region. An overproduction of the $A>140$ r-nuclei (relative to the solar distribution) could be obtained if a sizable part of the inner crust was ejected at the same time (Arnould et al. 2007). When the mass ejection is restricted to parts of the outer crust (up to a pressure value $P<P_{\text {drip }}$ ), different r-abundance distributions are obtained, as shown in Fig. 12. The production of r-nuclei in such a scenario is clearly sensitive to the thickness of the outer crust that is ejected and therefore depends on the ejection mechanism that is invoked. 
S. Goriely et al.: The r-process nucleosynthesis in neutron stars

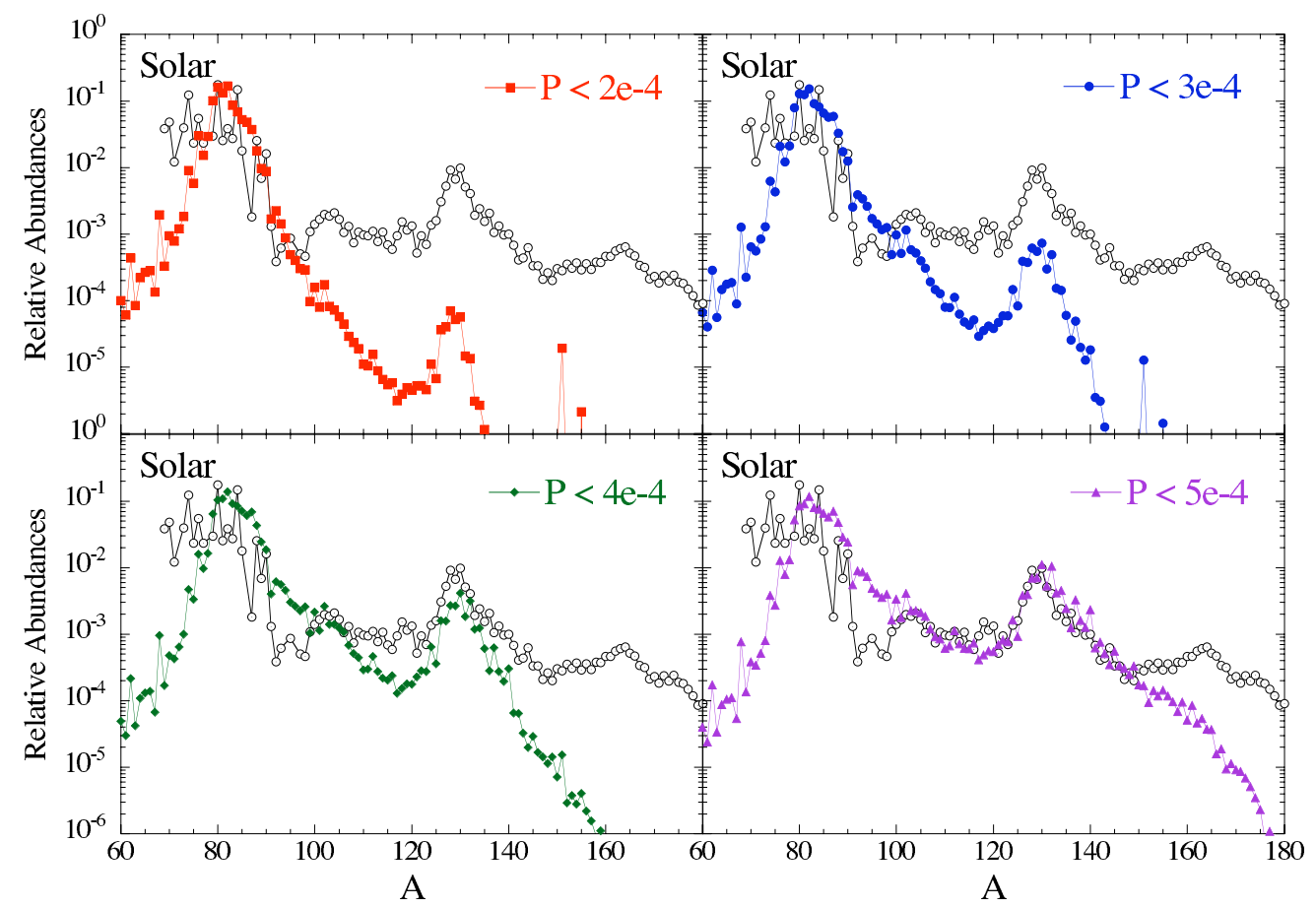

Fig. 12. Final abundance distributions for the whole outer crust (as integrated up to a pressure $P$ given in the legend in $\mathrm{MeV} / \mathrm{fm}^{3}$ ) after decompression when initially in NSE at $T_{9}=8$. The calculations are performed with the HFB-19 mass model and corresponding rates. The distributions are compared with the solar r-abundance distributions (dotted circles).

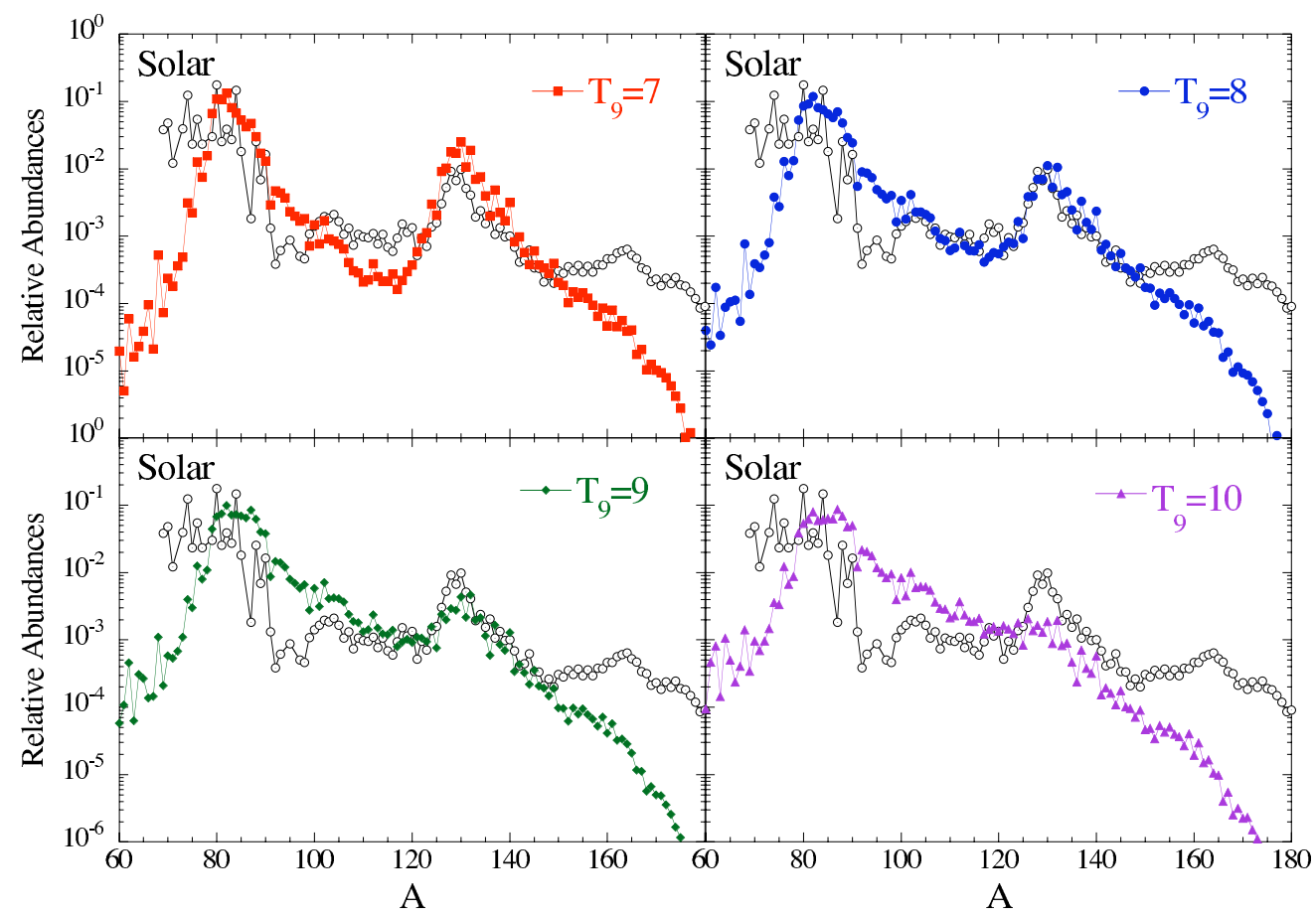

Fig. 13. Final abundance distributions of the outer crust material after decompression, if initially in NSE at different temperatures ranging between $T_{9}=7$ and $T_{9}=10$. The calculations are performed with the HFB-19 mass model and corresponding rates. The distributions are compared with the solar r-abundance distributions (dotted circles).

The overall abundance distribution depends not only on the fraction of the ejected crust but also on the initial temperature at which the NSE has been frozen in. We show in Fig. 13 the abundance distributions obtained for different initial temperatures between 7 and $10 \times 10^{9} \mathrm{~K}$, assuming that the whole outer crust is ejected.

It could be seen as purely fortuitous that temperatures around $T_{9} \simeq 8$ give rise to $\mathrm{r}$-abundance distributions in agreement with the solar distribution. However this is in agreement with the astrophysical scenario considered here. In particular, the temperatures considered here typically correspond to those at which NSE can be dynamically achieved in cooling events. We have calculated the typical timescale $\tau_{\text {NSE }}$ needed to reach NSE for a density of $\rho=3 \times 10^{11} \mathrm{~g} / \mathrm{cm}^{3}$ (typical of the most contributing part of the outer crust) and for different values of $Y_{\mathrm{e}}$ (as found in the outer crust). This timescale is obtained by estimating the time 


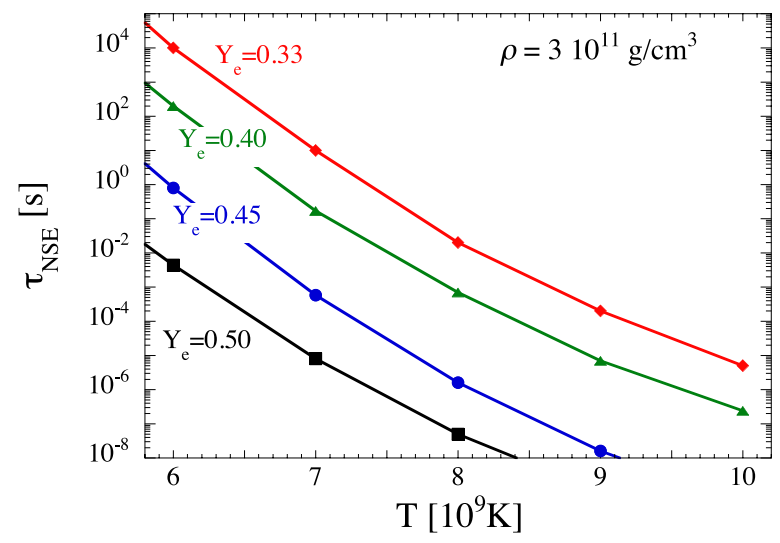

Fig. 14. Time needed to reach NSE as a function of the temperature for different values of $Y_{\mathrm{e}}$ at a constant density of $\rho=3 \times 10^{11} \mathrm{~g} / \mathrm{cm}^{3}$. Symbols corresponding to the same value of $Y_{\mathrm{e}}$ are connected by a solid line. $Y_{\mathrm{e}}=0.50$ is shown by squares, 0.45 by circles, 0.40 by triangles and 0.33 by diamonds.

needed to reach a steady state at constant temperature and density considering different initial seed nuclei for the chosen value of $Y_{\mathrm{e}}$. A full network including all strong and electromagnetic interactions (but no weak interactions) is used for this purpose. As shown in Fig. 14, for matter with initial values of $Y_{\mathrm{e}}=0.33-$ 0.40 , it takes about 1 to $20 \mathrm{~ms}$ to reach an NSE at $T_{9}=8$ and $\rho=3 \times 10^{11} \mathrm{~g} / \mathrm{cm}^{3}$, while at $T_{9} \simeq 9$, around $0.2 \mathrm{~ms}$ are required for the most neutron-rich conditions $\left(Y_{\mathrm{e}}=0.33\right)$. Such ms timescales are characteristic of dynamical scenarios of interest here for the potential mass ejection (e.g. in the bursts of soft gamma-repeaters, during NS mergers, ...), so that prior to the ejection the NSE should be achieved at temperatures typically above $T_{9} \simeq 8-9$ and can be expected to be frozen below during the ejection. The final production of r-nuclei is clearly sensitive to the temperatures at which NSE freezes in so that depending on the dynamical timescales different distributions (as seen in Fig. 13) are found.

Uncertainties in the nuclear physics input can influence the calculation of the final abundances. These concern essentially nuclear masses and the corresponding reaction rates. We show in Fig. 15 the abundance distributions obtained with NSE distributions and reaction rates determined on the basis of different mass models. The initial NSE temperature is $8 \times 10^{9} \mathrm{~K}$ and the whole outer crust mass is assumed to be ejected. As shown in Fig. 15, the exact strength, location, and width of the $N=50$ and $N=82$ r-process peaks are sensitive to the mass model adopted. The production of the $90 \lesssim A \lesssim 120$ r-nuclei can also be significantly modified according to the nuclear physics input. The importance of nuclear masses in this specific r-process nucleosynthesis scenario is essentially linked to the initial NSE conditions, which define not only the most abundant species initially present in the outer crust, but also the amount of free neutrons available for the neutron-capture process during the decompression. In contrast, the impact of uncertainties affecting the $\beta$-decay rates is found to be rather limited (as illustrated in Fig. 16), though $\beta$-delayed neutron emission plays some role in smoothing the final abundance pattern.

\section{Conclusion}

Most of the problems faced in understanding the origin of $r-$ process elements and observed r-abundances are related to our ignorance of the astrophysical site that is capable of providing the required high neutron flux. We have shown here that the

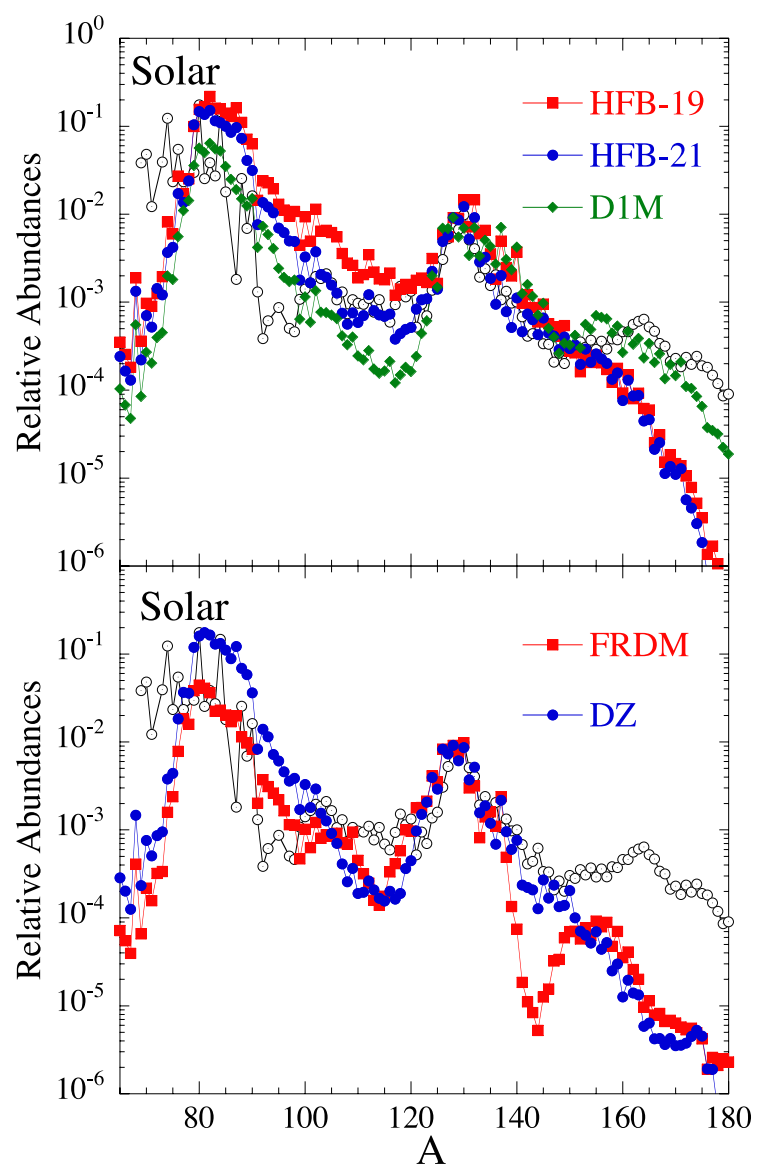

Fig. 15. Same as Fig. 13 for an initial NSE temperature of $T_{9}=8$ and five different mass models (and corresponding reaction rates), namely the HFB-19, HFB-21 and D1M mass models (upper panel) and the Duflo \& Zuker (DZ; 1995) and FRDM (Möller et al. 1995) models (lower panel).

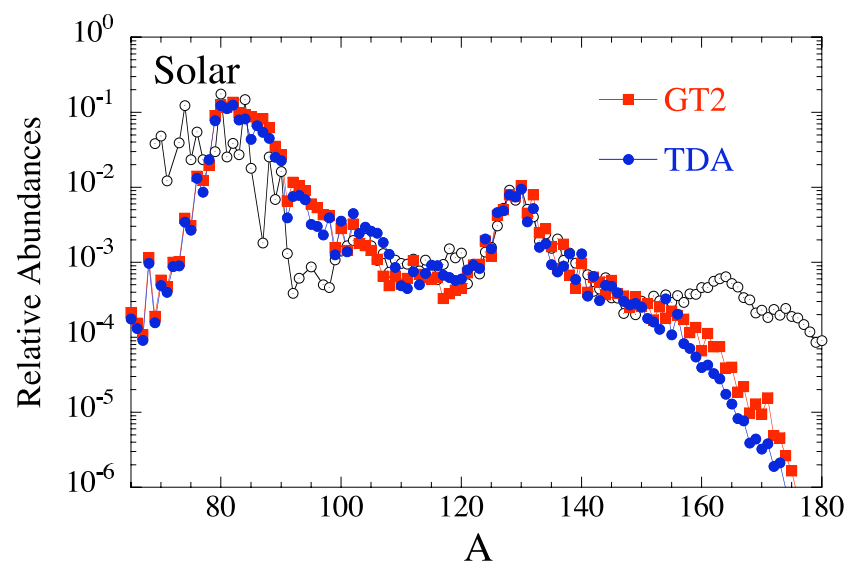

Fig. 16. Same as Fig. 13 for an initial NSE temperature of $T_{9}=8.5$ and two different calculations of the $\beta$-decay rates, namely the Gross Theory (version 2; Tachibana et al. 1990) and the Tamm-Dancoff approximation (TDA) of Klapdor et al. (1984). The calculations were performed with the HFB-21 mass model and corresponding rates.

decompression of the NS matter from the outer crust provides suitable conditions for a robust r-processing of the light species, i.e., r-nuclei with $A \leq 140$, provided the outer crust is initially in NSE at temperatures around $T_{9} \simeq 8$ and the ejection mechanism allows for the full outer crust to be expelled to the interstellar 
medium. During the decompression, the free neutrons (initially liberated by the high temperatures) are re-captured leading to a final pattern similar to the solar system distribution.

While the total mass included in the outer crust only amounts to some $10^{-5}-10^{-4} M_{\odot}$, its ejection leads to a galactic enrichment in stable neutron-rich nuclei with a final composition close to what is now observed in the solar system. The final composition should carry the imprint of the initial temperature at which the NSE is frozen in prior to the ejection, as well as the density region of the outer crust that gets ejected. The final abundances are also affected by nuclear uncertainties, most particularly nuclear masses. The similarity between the predicted and solar abundance patterns as well as the robustness of the prediction against variations of input parameters (such as expansion timescales or initial $Y_{\mathrm{e}}$ distribution) make this site one of the most promising that has been proposed, deserving further exploration with respect to various aspects such as nucleosynthesis, hydrodynamics, and galactic chemical evolution.

Acknowledgements. The authors are thankful to D. G. Yakovlev and A. Y. Potekhin for valuable discussions. S.G. and N.C. acknowledge the financial support of the "Actions de recherche concertées (ARC)" from the "Communauté française de Belgique". S.G. and N.C. are FNRS research associates. H.T.J. acknowledges support by the Deutsche Forschungsgemeinschaft through the Transregional Collaborative Research Centers SFB/TR 27 "Neutrinos and Beyond" and SFB/TR 7 "Gravitational Wave Astronomy", and the Cluster of Excellence EXC 153 "Origin and Structure of the Universe" (http://www. universe-cluster.de). J.M.P. thanks the NSERC (Canada) for financial support.

\section{References}

Arcones, A., Martinez-Pinedo, G., Roberts, L. F., \& Woosley, S. E. 2010, A\&A, 522, A25

Argast, D., Samland, M., Thielemann, F.-K., \& Qian, Y. 2004, A\&A, 416, 997
Arnould, M., Goriely, S., \& Takahashi, K. 2007, Phys. Rep., 450, 97 Audi, G., Wapstra, A. H., \& Thibault, C. 2003, Nucl. Phys. A, 729, 337 Audi, G., Wang, M., Wapstra, A. H., Pfeiffer, B., \& Kondev, F. G. 2010, Priv. Comm.

Baym, G., Bethe, H. A., \& Pethick, C. J. 1971, Nuc. Phys. A, 175, 225

Bravo, E., \& Garcia-Senz, D. 1999, MNRAS, 307, 984

DeWitt, H., Slattery, W., \& Chabrier, G. 1996, Physica B, 228, 21

Duflo, J., \& Zuker, A. P. 1995, Phys. Rev. C, 52, R23

Fischer, T., Whitehouse, S. C., Mezzacappa, A., Thielemann, F.-K., \& Liebendrfer, M. 2010, A\&A, 517, A80

Glazyrin, S. I., \& Blinnikov, S. I. 2010, J. Phys. A: Math. Theor., 43, 075501

Goriely, S., Demetriou, P., Janka, H.-Th., et al. 2005, Nuc. Phys. A, 758, 587c

Goriely, S., Hilaire, S., \& Koning, A. J. 2008, A\&A, 487, 767

Goriely, S., Hilaire, S., Girod, M., \& Péru, S. 2009, Phys. Rev. Lett., 102, 242501

Goriely, S., Chamel, N., \& Pearson, J. M. 2010, Phys. Rev. C, 82, 035804

Haensel, P., \& Pichon, B. 1994, A\&A, 283, 313

Haensel, P., Potekhin, A. Y., \& Yakovlev, D. G. 2007, Neutron Stars 1, Astrophys. Space Sci. Lib., 326 (Springer)

Hüdepohl, L., Müller, B., Janka, H.-T., Marek, A., \& Raffelt, G. G. 2010, Phys. Rev. Lett., 104, 251101

Janka, H.-T., Eberl, T., Ruffert, M., \& Fryer, C. L. 1999, ApJ, 527, L39

Klapdor, H. V., Metzinger, J., \& Oda, T. 1984, At. Data Nucl. Data Tables, 31, 81

Lattimer, J. M., Mackie, F., Ravenhall, D. G., \& Schramm, D. N. 1977, ApJ, 213, 225

Meyer, B. S. 1989, ApJ, 343, 254

Möller, P., Nix, J. R., Myers, W. D., \& Swiatecki, W. J. 1995, At. Data Nucl. Data Tables, 59, 185

Nadyoshin, D. K., \& Yudin, A. V. 1999, MNRAS, 307, 984

Oechslin, R., Janka, H.-T., \& Marek, A. 2007, A\&A, 467, 395

Pearson, J. M., Goriely, S., \& Chamel, N. 2011, Phys. Rev. C, in press

Pethick, C. J., \& Ravenhall, D. G. 1995, Ann. Rev. Nucl. Part. Sci., 45, 429

Pothekin, A. Y., \& Chabrier, G. 2000, Phys. Rev. E, 62, 8554

Rosswog, S., Speith, R., \& Wynn, G. A. 2004, MNRAS, 351, 1121

Rüster, S. N., Hempel, M., \& Schaffner-Bielich, J. 2006, Phys. Rev. C, 73, 035804

Sumiyoshi, K., Yamada, S., Suzuki, H., \& Hillebrandt, W. 1998, A\&A, 334, 159

Tachibana, T., Yamada, M., \& Yoshida, Y. 1990, Prog. Theor. Phys., 84, 641

Timmes, F. X., \& Arnett, D. 1999, ApJS, 125, 277

Tsuruta, S., \& Cameron, A. G. W. 1965, Can. J. Phys., 43, 2056 